KOMMENTARE UND BERICHTE

\title{
Situation der Philosophie, Kultur der Philosophen Über die neudeutsche Universitätsphilosophie
}

\section{von ULRICH JOHANNES SCHNEIDER (Leipzig)}

Seit 199() sind westdeutsche Verhältnisse auch im Osten der erweiterten BRD anzutreffen: Für wenige Bereiche trifft das so allgemein zu wie für die Universitäten, die in den neuen Bundesländern strukturell denen der alten Bundesländer gleichgemacht wurden. Die Inhalte haben gewechselt. je nach Disziplin. Aber ist damit alles gesagt? Was heißt das zum Beispiel für die Philosophie. nachdem die Universitäten das westdeutsche Muster adaptiert haben. und zugleich Inhalte und Personen fast völlig ausgetauscht wurden?

In der DDR gab es Philosophie als Fach an mehreren Universitäten in eigenen Sektionen. die neben den Sektionen für Marxismus-Leninismus bestanden. Zwar wurden alle Lehrenden der Philosophie als Marxisten-Leninisten angesehen und verstanden sich in der Regel selbst auch so, es gab gleichwohl ein relativ breites Spektrum verschiedener Interessen, sowohl in historischer als auch in systematischer Hinsicht. Wie im Westen gab es Philosophiehistoriker für Antike. Mittelalter. Neuzeit und Moderne (genannt ,bürgerliche Philosophie“). dazu noch solche für dic zeitgenössische westliche Philosophie (genannt "spätbürgerliche“" Philosophie). Im systematischen Bereich variierten die Interessen von Erkenntnistheorie über Wissenschaftstheorie bis hin zu ethischen und sozialen Problemen. Es gab in der Forschung durchaus so etwas wie die Bildung schulähnlicher Zusammenhänge um einzelne Lehrstühle.

\section{Allseitiges Schweigen}

Heute sind die Interessen derer, die an den Universitäten der neuen Bundesländer Philosophie unterrichten, ungleich stärker diversifiziert. Das hat vor allem damit zu tun, daß der Marxismus-Leninismus keine Rolle mehr spielt. Mit der Staatsideologie ist in Ostdeutschland - anders als in Rußland oder Polen - auch der dazugehörige philosophische Betrieb verschwunden; was bis 199() die Geister beschäftigte, wurde in kurzer Zeit zu einer Größe, über die man nur in der Vergangenheitsform sprechen kann. Dazu kommt, daß die Protagonisten der Philosophie in der DDR heute keine Stimme mehr haben: Während

"Teile des folgenden Textes sind identisch mit einem unveröflentlichten Meinungsbild. das der Verfasser alul Anfrage der UNESCO erstellt hat. Der Verfasser. aus dem Westen Deutschlands kommend und scit 1992 Assistent am Philosophischen Institut der Universität Leipzig. hat seine Ausührungen mit viclen ost- und westdeutschen Philosophen diskutiert, meistens kontrovers. Vielleicht kann diese Veröffentlichung dazu dienen. das im Titel angesprochene Thema zu vertiefen. 
Schriftsteller aus der DDR öffentlich sich noch verteidigen oder erklären, was sie früher schrieben oder sagten, schweigen die Philosophen - mit wenigen Ausnahmen (vgl. die Beiträge in Kapferer (1994)). Sie werden auch nicht gefragt. Zu ihrem Elend kommt noch. daß im Moment ihres Funktionsverlusts auch schon ihre Geschichte geschrieben schien von westdeutschen Autoren (vgl. Wilharm und Kapferer (1990)).

Etwas unheimlich mutet an, daß diese Zerstörung einer philosophischen Kultur durch eine andere, diese Ersetzung einer Philosophie durch eine andere die Situation der Philosophie im Grunde nicht verändert hat. Heute wird die Philosophie von den Regierungen der einzelnen Länder ebenso relativ großzügig ..eingerichtet" und ...ausgestattet" wie zuvor im sozialistischen Zentralstaat die philosophischen Sektionen. Aber auch diese administrative Stützung ist ein schweigend vollzogenes Werk. Die Universitätsphilosophie scheint aus einer Selbstverständlichkeit in die andere zu geraten. ohne daß eine nicht bloß lokale Diskussion darüber stattgefunden hätte. Der funktionierende Philosophiebetrieb von heute ist somit in ein dreifaches Schweigen eingebettet: erstens das der ehemaligen DDRPhilosophen. die historisch marginal geworden sind; zweitens das der neuen Regierungen. die mit politischen Mitteln neue Strukturen schaffen: und drittens das derjenigen, die auf den freigewordenen Stellen berufen sind - und dieses Schweigen ist vielleicht am beunruhigendsten, weil es ein Schweigen über die herrschenden Verhältnisse ist.

Wenn westdeutsche Professoren .,im Osten“ auftreten, als Teilnehmer an Kolloquien und Workshops, dann wissen sie, daß sie einem Teil ihres Publikum fremd sind. Das halten sie aber aus, hoffend vielleicht auf Gewöhnung und die Macht der Verhältnisse. Nur sehr selten lenken sie das Gespräch an den neueingerichteten Philosophie-Instituten der östlichen Länder auf den gesellschaftlichen Umbruchprozeß und dessen Bedeutung für ihre Disziplin. Meist wird eine Eingangsbemerkung dazu gemacht. Das akademische Reden und Vortragen scheint den meisten Universitätsphilosophen so sehr Selbstzweck geworden zu sein. daß sie sich über die Bedingungen ihres Verstandenwerdens keine Gedanken mehr machen. Sie haben an anderen Orten schon geübt, die Zuhörer nur als Publikum zu nehmen, als Staffage ihrer Eitelkeit. Die Baustellen in den ostdeutschen Innenstädten beeindrucken viele. aber für die ..Umbauarbeiten im Kopf“ der Menschen dort interessiert man sich selten. Den meisten scheint, schlimmer noch, das eine wie das andere ein äußerlicher Prozeß zu sein. Es ist eine Frage, welche die bezahlten Philosophen sich offenbar noch nicht richtig gestellt haben: die Frage nach der Bedeutung, die der administrativ erfolgreiche Umbau der Universitäten in den neuen Bundesländern für die Philosophie hat. Dabei ist kaum eine andere Disziplin in ihrer alten Form so gründlich abgebaut und unmittelbar zugleich damit so grundlegend aufgebaut worden wie die Philosophie an den Universitäten der neuen Bundesländer. Für die neu in den östlichen Bundesländern lehrend und forschend Tätigen war ein Universitätswechsel nichts Unerwartetes, sondern im Gegenteil ein gewöhnlicher Karriereschritt. Wenn man die Sprache des Beamtenrechts verwenden will, handelte es sich für die an westdeutschen Universitäten ausgebildeten Philosophen um eine ..Beförderung“. Beförderung kann man auch allgemein verstehen: Befördert wurden nicht nur Personen, sondern auch Ideen. Haltungen. Auffassungs- und Vermittlungsweisen usw., die mit den in der westdeutschen Gesellschaft sozialisierten Personen verbunden sind. Entlassen dagegen wurde nicht nur eine große Zahl von Personen, die vor 1990 an der Universität angestellt waren. sondern auch eine ganze Reihe von Ideen. Haltungen. Auffassungs- und Vermittlungsweisen usw.. die mit den in der ostdeutschen Gesellschaft sozialisierten Personen verbunden waren.
Die Situation der Philosophie in den Jahren nach dem Oktober 1990 ist allgemein durch diese beiden Größen gekennzeichnet: Beförderung und Entlassung. In den neuen Bundesländern bestimmen diese Größen auch individuelle Schicksale: Nachdem diese und jene Personen entlassen, d. h. aus der Universität ausgeschlossen waren, wurden diese und jene anderen Personen auf die freien Stellen befördert. Beides hat Zeit in Anspruch genommen, was bei allen ..Beteiligten“ (Beförderten und Entlassenen) die Nerven angriff. Inzwischen hat sich die Spannung gelöst, die Konfrontation zwischen Altem und Neuem ist entschieden, zumindest was den Personalbestand angeht.' Es drängt sich folgender Eindruck auf: Die Beförderung von Ideen und Gedanken .,westlicher Prägung“ an die Institute und Hörsäle ..im Osten“ ist von allen Beteiligten als ein Bäumchen-Wechsel-DichSpiel aufgefaßt und akzeptiert worden, dessen Regie den Landesregierungen niemals streitig gemacht wurde. Die Ersetzung der Philosophie durch Philosophie ging so reibungslos vonstatten. weil es Politikern überlassen wurde, diese Ersetzung zu bewerkstelligen, und Politiker haben der Einfachheit halber Köpfe ausgetauscht. Eine philosophische Diskussion über die gesellschaftliche Funktion oder Situation der Philosophie konnte ihnen dabei nicht in die Quere kommen - diese Diskussion gab es schon im geteilten Deutschland auf keiner Seite. Philosophen waren Staatsdiener hüben wie drüben und sind es noch heute; ganz wie Soldaten oder Polizisten bequemen sie sich Regeln und Gesetzen; das Befolgen und Gehorchen gelingt ihnen von allein.

Vor 1990 waren sich die Philosophen der BRD und der DDR weitgehend unbekannt. Nur privilegierte DDR-Akademiker konnten an Tagungen im Westen teilnehmen; im Westen bestand meist nur wenig Interesse an Philosophen aus der DDR. Es waren in der Mehrzahl Wissenschaftstheoretiker und Philosophiehistoriker der DDR, die im Westen persönlich bekannt waren. Als 1992 eines der führenden Mitglieder des ehemaligen ..Zentralinstituts für Philosophie" an der Akademie der Wissenschaften der DDR gefragt wurde, was denn von der .,DDR-Philosophie“" seiner Meinung nach bleiben würde, antwortete er zur Überraschung vieler: die großen Gesamtausgaben. In der Tat sind die teilweise in Ost-West-Zusammenarbeit unternommenen Werkausgaben von Aristoteles und Leibniz gute Beispiele für den Minimalkonsens, auf dem die gegenseitige Anerkennung auch vor 199() beruhte.

1 Bis 1990) lehrten etwa 30) Universitätsprofessoren Philosophie in der DDR: es gab an 5 Universitäten eigenc ...Sektionen für marxistisch-leninistische Philosophie“ (2 große Scktionen in Berlin und Leipzig mit mehr als 10) Professoren. 3 kleine in Greifswald. Halle und Jena). An der Universität Dresden konnten Studenten im Fernstudium Philosophie studieren. an anderen Hochschulen gab es einzelne Lehrstühle für Philosophie. Zusammen mit Assistenten kann man die Zahl der akademischen Lehrer der Philosophic in der DDR auf ca. 1.5() schätzen. Etwa chensoviele Philosophen waren an diversen Forschungseinrichtungen tätig (also nicht in der Lehre). die meisten davon im ..Zentralinstitut für Philosophie" der ..Akademic der Wissenschaften der DDR“. Heute gibt es 12 philosophische Institute an den Universitaten in Mecklenburg-Vorpommern (Rostock und Greifswald). Brandenburg (Cottbus. Frankfurt an der Oder und Potsdam). Sachsen-Anhalt (Halle und Magdeburg). Thüringen (Jena und Erfurt) und in Sachsen (Leipzig. Chemnitz und Dresden). Die Humboldt-Universität im Land Berlin zählt chenfalls zu den umstrukturierten Universitäten. An allen Instituten zusammen lehren ca. 35 Philosophieprofessoren (in Berlin und Dresden mehr als 4 pro Institut. sonst weniger): dic Zahl der Assistenten und anderer Lehrkräfte ist höher (ca. 60). Insgesamt lehren 1995 in Deutschland an ca. 70) Universitäten ca. 330) Philosophieprofessoren (ohne Assistenten und andere Lehrkräfte). 
Mit anderen Worten: Es gab keine fachliche Auseinandersetzung, eine Diskussion etwa zur Bedeutung des Marxismus fand auf einer ost-westdeutschen Ebene nicht statt (Ausnahmen bestätigen die Regel). Das ist keineswegs erstaunlich. denn es gilt auch für die Verhältnisse zwischen Frankreich und Deutschland, zwischen England und Deutschland, zwischen Frankreich und England. daß man auf jeder Seite die Denker aus dem jeweils anderen Land oft für befangen, ja für voreingenommen hält. Warum sollte es zwischen den beiden deutschen Staaten anders gewesen sein? Bis auf einzelne marxistische Wissenschaftler im Westen interessierte sich niemand für die Diskussionen im Osten, weil man kein Interesse hatte, sich einzumischen. Genauso umgekehrt: An den Lehrstühlen für "Geschichte der bürgerlichen Philosophie“ wurden die philosophischen Debatten Westdeutschlands zwar kommentierend begleitet. nicht aber mit der Absicht. daran teilzunehmen. Die im politischen Bereich einigermaßen „, friedliche Koexistenz“ der beiden deutschen Staaten wurde im kulturellen und wissenschaftlichen Bereich durch Ahnungslosigkeit und Desinteresse, auch durch ein implizites Überlegenheitsgefühl gestützt - auf beiden Seiten.

Das Kommunikationsdefizit vor 199() hatte natürlich etwas mit der politischen Lage zu tun. aus der auch die Philosophie begriffen werden muß. Philosophieprofessoren waren beiderseits der deutschen Mauer - wenn auch in verschiedenem Grade merklich - ziemlich direkt mit dem Staat verbunden. in dem sie lebten. Im Osten waren die meisten Philosophen Mitglieder der SED und daher über Versammlungen und Aktivitäten anderer Art mit den Meinungsbildungsprozessen der Partei- und Staatsführung vertraut. Nur zur Stunde der mehrmals im Monat regelmäßig stattfindenden Parteiversammlung seien alle Universitätsbüros besetzt gewesen, sagen die Sekretärinnen. Die Universität selbst war der Ort der engsten Berührung von Partei-Ideologie und akademischer Tätigkeit - die im engeren Sinn wissenschaftliche Arbeit fand zu Hause und in Bibliotheken statt.

Westlich der Mauer gab es keine direkte Kontrolle der Aktivitäten der Philosophieprofessoren, sie waren aber, wie alle Professoren. Beamte. und mußten mit dem Eid auf die Verfassung etwa in Westberlin auch das Versprechen ablegen. Kontakte mit Personen aus der DDR einer staatlichen Stelle anzuzeigen. Der Augenblick der Verbeamtung war hier (und ist noch) die letzte entscheidende Hürde vor dem Professorenstatus, den man danach eigentlich nicht mehr verlieren kann. Die als Philosophieprofessoren gescheiterten Arthur Schopenhauer und Friedrich Nietzsche haben bereits im 19. Jahrhundert Spott über die staatliche Anstellung .,freier“ Denker vergossen. In der Tat findet die Lehre und Forschung an deutschen Universitäten seit je die äußerste Grenze ihrer Freiheit in der ministerialen Personalpolitik und den darauf orientierten Rücksichten.

\section{Die akademische Philosophie: neuen und alten Mächten ausgeliefert}

Die Abhängigkeit der Philosophieprofessoren von der Regierung besteht ganz einfach darin, daß die Personalpolitik nicht in ihren Händen liegt, sondern in denen eines Ministers, der Professoren beruft. Bei den neuen Bundesländern kommt hinzu. daß hier die Personalpolitik weniger Berufungs- als Abberufungspolitik ist.

Das Ministerium setzt neue Leute durch: Das gilt im deutschen System ganz generell und war auch beispielsweise bei Ernst Bloch so, der seine erste Professur 1949 in Leipzig gegen das Votum der Universität nur dank des damaligen Sächsischen Ministeriums erhielt. Nach
1990 haben die Ministerien der neuen Länder die Flut der Besetzungsfragen durch eigens gebildete Fachkommissionen zu bewältigen versucht, die hauptsächlich aus westdeutschen Professoren bestanden mit nur einigen wenigen (zahlenmäßig nicht entscheidenden) ...alten" Mitgliedern der jeweiligen Universität. Inzwischen sind alle Institute besetzt, und es haben drei oder vier DDR-Philosophen den Sprung auf Professorenstellen „,neuen Rechts“ geschafft, einigen jüngeren Wissenschaftlern könnte es in Zukunft gelingen. Die Durchsetzung des Neuen stand seitens der Ministerien immer im Zeichen der Abwehr des Alten; der Hebel war hier die Logik der Stellenbesetzung. die eine Logik des Entweder-Oder ist. Unvermeidlich war in ministerieller Perspektive die Entlassung von DDR-Philosophieprofessoren Voraussetzung der Neubesetzungen. DDR-Philosophen verloren ihre Arbeit fast alle aus politischen Gründen, die im ..Einigungsvertrag" zwischen der BRD und der DDR in juristische Kriterien verwandelt wurden. Ihre wissenschaftliche und pädagogische Qualifikation war nie Thema. In Leipzig gibt es drei Fälle. wo verdiente und über die Grenzen der DDR hinaus bekannte und anerkannte Philosophieprofessoren nicht mehr als Lehrende oder Forschende mit der Universität in engere Verbindung treten können. Die Macht des Ministeriums bewirkte eine radikale Abtrennung des Alten vom Neuen. Nur wenige der Geschaßten haben die philosophische Gelassenheit. persönliche und wissenschaftliche Kontakte über den Graben hinweg zu knüpfen.

Der Einigungsvertrag nennt die Schließung ganzer Einrichtungen ..Abwicklung“. Auch die Umgestaltung und Umbesetzung der Sektionen für Philosophie (jetzt Institute für Philosophie) wurde Abwicklung genannt: ..wegen Abwicklung“" wurde gekündigt (..mangels Bedarf" sagte dasselbe mit anderen Worten). Die Humboldt-Universität Berlin hat früh schon gegen dieses Verfahren geklagt und gegen das Land Berlin Recht erhalten: Eine Auflösung nur von Teilen (Instituten) oder gar nur eine Neubesetzung von freigemachten Stellen sei keine Abwicklung: jede Kündigung erfordere deshalh eine ..Begründung im Einzelfall“. In Leipzig wurden sogar einige Sekretärinnen, die keinen Tag zu arbciten aufgehört hatten. wegen ..Abwicklung“ der Sektion für Philosophie und Neugründung des Instituts für Philosophie ohne Anrechnung der abgeleisteten Dienstjahre von einem Tag auf den anderen als neu angestellt cingestuft. Wie in Sachsen, so erhalten auch in anderen Ländern die meisten wegen Abwicklung oder ähnlich pauschalen Gründen Gekündigten vor Gericht Recht, ohne damit schon in ihre alten Arbeitsverhältnisse zu gelangen.

Das durch solche Willkürmaßnahmen geprägte inneruniversitäre Klima war einer Diskussion der Neuorganisation der Universitäten natürlich nicht förderlich. Im Grabenkampf um Stellen und um eine gerechte Beurteilung ihrer früheren Tätigkeit sind viele DDR-Philosophen verbittert und haben die Energie verloren, sich an einer wissenschaftlichen Auseinandersetzung zu beteiligen. Aber auch auf seiten der neubestallten Wissenschaftler ist die regierungsamtliche Radikalität auf keinen nennenswerten Widerstand gestoßen. Der eklatante Mangel an Reflexion über die Stellung der Philosophie in der Gesellschaft hat ostwie westdeutsche Philosophen zu Schachfiguren einer Politik werden lassen. die auf das reibungslose Funktionieren von Lehre und Forschung abzielt. Eine ..funktionierende Universität" wird in öffentlichen Erklärungen von Universitätsrektoren und Universitätspräsidenten oft gefordert. wobei man sich aber darunter offensichtlich vor allem eine Universität ohne politische Auseinandersetzung vorstellt.

Die durch die Kultusministerien verantwortete Personalpolitik hat in Deutschland eine 
lange Tradition: gleichwohl ist das widerspruchslose Funktionieren dieser Politik in der Zeit nach $199($ ) besonders auffällig. Genauso auffällig ist das unkommentierte Hinnehmen einer ganz anderen Personalpolitik, die von mehr oder weniger geheimen Kommissionen gestaltet wurde, und denen die Universitätsinstitute als Einrichtungen des Öffentlichen Dienstes ausgeliefert sind. Ihre Mitglieder, vor allem diejenigen. die vorher in der DDR arbeiteten, waren der Beurteilung im Hinblick auf eine Mitarbeit bei der .,Stasi“ ausgesetzt. Nach allem, was bekannt geworden ist, finden sich in den Beurteilungen. die von einigermaßen willkürlich zusammengesetzten und selbst unkontrollierten Personalkommissionen zur Entscheidung befördert werden. moralische und juristische Kriterien gemischt. Mit anderen Worten: Es werden bestimmte Arten von Tätigkeiten aus der Zeit vor 199() gegen einzelne Personen gewendet, ohne daß die Kriterien der Entscheidungen oder die Qualifikation der Entscheidenden eingehend diskutiert würde. Im Bereich der Philosophie vollzieht sich nicht anders als anderswo eine schleichende ..Abrechnung" mit der DDR. wo fast alles undurchsichtig bleibt: Täter, Gründe, Opfer.

Der Stasi-Vorwurf scheint ganz einfach zu sein: Wer irgendwann einmal mit dem Ministerium für Staatssicherheit der DDR zusammengearbeitet hat, offiziell oder inoffiziell darf kein Beamter werden bzw. im Bereich des Öffentlichen Dienstes keine Anstellung erhalten. Eine inzwischen langjährige öffentliche Debatte um diese allgemeine Strategie der neudeutschen Politik (und um einige wenige Einzelfälle) hat dazu geführt. heute viele Formen der ..Zusammenarbeit" als eher harmlos anzusehen: Kritik hat sich artikuliert an der Benutzung von Akten eines Ministeriums, in dem Fälschung und Desinformation geübte Praxis war. Das Problem ist sehr komplex, denn es gab auch Standpunkte wie den des Schriftstellers Heiner Müller, der zugab, mit der ..Stasi“ Kontakte gepflegt zu haben, weil ihm bewußt gewesen sei. mit den Leuten der Partei - besonders in den letzten Jahren der DDR - nicht mehr reden zu können. Ein philosophischer Mitarbeiter aus Leipzig. der ähnlich argumentierte und seine Zusammenarbeit mit der Stasi frühzeitig öffentlich machte. weil er so seine Unschuld beweisen wollte, erreichte damit nur seine sofortige Entlassung. Die meisten reagierten und reagieren anders und warten ab, bis die Mitarbeiter der GauckBehörde fündig werden. Wiederum in Leipzig sind 1993 zwei Kollegen von einem auf den anderen Tag verschwunden - sie wurden ,.rausgegauckt“. wie man sagt. Nichts an diesem Proze $ß$ der Beurteilung war öffentlich: demokratisch nicht legitimierte Personalkommissionen auf der Ebene der Universitäten und der Landesregierungen führten geheime Verfahren zur Anhörung der Betroffenen und Urteilsfindung durch. Den Entlassenen blieb der Weg der Klage, den viele erfolgreich beschritten. ohne allerdings eine Wiedereinstellung erreichen zu können. In keinem Fall hat eine Beurteilung der konkreten Art der Zusammenarbeit die Entscheidung beeinflußt. es war immer nur die Tatsache der Zusammenarbeit selbst ausschlaggebend.

Inzwischen sind die Akten der Stasi von und über Philosophen weitgehend erforscht und vom zuständigen Mitarbeiter der Gauck-Behörde jüngst in groben Zügen öffentlich bekannt gemacht worden (vgl. Herzberg (1994)). Daraus kann man entnehmen, daß die Stasi nur selten rein inhaltliche Gründe hatte, Informationen über Philosophen zu sammeln oder gar in deren Karriere einzugreifen - auch wenn solche inhaltlichen Gründe von Mitarbeitern, d. h. von Philosophen-Spitzeln. angedient wurden. Interesse bestand im wesentlichen an der allgemeinen ideologischen Zuverlässigkeit und an Informationen über Kontakte einzelner Personen ins westliche Ausland. In merkwürdig ähnlicher Fortsetzung dieses
Auskundschaftens wissenschaftlich irrelevanter Daten arbeiten die Personalkommissionen im Verein mit der Gauck-Behörde heute noch an einer schematischen Klassifizierung der in der DDR sozialisierten Menschen in ..Täter" und ..Opfer”. indem sie die bloße Dokumentierung einer Mitarbeit zur Basis einer Verurteilung machen. So wurde beispielsweise 1993 ein Mitglied des Leipziger Instituts für Philosophie vor die universitäre Personalkommission zitiert. die ihm zwei neuaufgefundene Dokumente zur Last legte: ein Schreiben der Stasi. worin dem Mitarbeiter eine Auslandsreise untersagt wurde mit der Begründung. es sei zu einem früheren Zeitpunkt nicht zu der gewünschten Zusammenarbeit gekommen. und einen leeren Ordner. der offenbar für Berichte angelegt worden war, die der Betreffende nicht geschrieben hat. Die Vermutung ist nicht von der Hand zu weisen. daß erst die Tatsache, daß der Betroffene diesen Vorgang im kleinen Kreis herumerzählte, ihn vor Konsequenzen bewahrt hat. die die Personalkommission aus den ja offenbar entlastenden Dokumenten gegen ihn zu folgern bereit gewesen wäre.

\section{Die Kultur der Philosophen: Verdächtigungen und blinder Fleck}

Mit der Demokratisierung der politischen Verhältnisse auf dem Gebiet der ehemaligen DDR hat sich nicht nur der Inhalt der Lehre der Philosophen verändert, sondern mit den westdeutsch gestalteten Universitäten wurde auch eine neue Situation für die Philosophie überhaupt geschaffen. Es gibt einen neuen Rahmen für Handeln und Verhalten der Universitätsphilosophen. Dieser Rahmen scheint nun tatsächlich weit weniger dem gegenseitigen Kennenlernen zu dienen als der Wucherung von Verdächtigungen. Politisch hat den größten Nachdruck der Verdacht gegen die DDR-Philosophen als sogenannte ...Kaderphilosophen“ (vgl. Kapferer (1990)), d. h. als Philosophen im Dienst der SED. Dieser Verdacht ist pauschal nicht einmal falsch. Er erklärt nur gar nichts, wenn man sich nicht (nachträglich bzw. rückblickend) in die Bedingungen hineinzudenken versucht, unter denen Studium und Forschung im sozialistischen Staat standen.

Für den westlichen Kopf ist das Nachplappern von partei-erzeugten Phrasen und die an der Oberfläche des Gedruckten nicht entdeckbare Kritik schon Zeichen von ..Kaderphilosophie“. für das östliche Selbstverständnis dagegen sind im Rückblick die Grenzen enger und pragmatischer gezogen. Kaderphilosophie im eigentlichen Sinn wird hier gar nicht zugegeben, wohl aber das Vorhandensein von Kaderphilosophen: Wissenschaftler, die sich die engeren und kurzfristigeren politischen Zielsetzungen der führenden Partei zu eigen machten und ihre philosophische Kritikfähigkeit dem politischen Kampf unterordneten. Die Überzeugung, daß gegen den Klassenfeind auch das wissenschaftliche Denken parteilich sein müsse. hat solche Philosophen im Vorwort immer zu wenigstens einem Lenin-Zitat greifen lassen: Wer das nicht tat, vielleicht sogar Marx und Engels anzuführen vermied, der war sicher kein Kaderphilosoph. Aber auch solche, die sich auf die .,Klassiker“ des Marxismus-Leninismus beriefen, taten es nicht immer blind und affirmativ. Die Kultur der Anspielungen und Auslassungen, der Abstufung von Haupt- und Seitenbemerkungen ist heute in ihrer DDR-spezifischen Raffiniertheit überflüssig geworden. Gleichwohl definierte diese Kultur wesentlich das Feld der intellektuellen Tätigkeit in der DDR-Philosophie.

Der verdächtigende Blick aus dem Westen auf die mehr oder weniger expliziten Zeichen der politischen Indienststellung des philosophischen Denkens in der DDR ist nicht sehr 
geübt. schon weil er zu selten als reflektierte Bemühung auf die eigene Arbeit angewand wurde. Daß er insgesamt mehr verstellt als erhellt. läßt sich u. a. daran erkennen. daß e nicht zu erklären vermag, wie in der DDR-Philosophie Konflikte entstanden und ausgetragen wurden. Daß es in der DDR der sechziger Jahre den Versuch gab, die jugoslawische Praxis-Philosophie zu diskutieren. daß es in den achtziger Jahren eine Debatte über Hei degger gab. daß .,bürgerliche Philosophie“ überhaupt zunehmend jenseits von Schablonen rezipiert wurde, das verweist auf einzelne Köpfe, die die Enge der philosophischen Debatten aufzubrechen gewillt waren. Daß die DDR-Philosophie dennoch bis zum Ende enger und langweiliger blieb als etwa die russische oder polnische Philosophie. kann man zwar behaupten - und sicher mit ähnlichem Recht. mit dem man die ..BRD-Philosophie“ der letzten Jahrzehnte enger und langweiliger finden kann als etwa die französische oder amerikanische Philosophie - , es heißt aber noch nicht, die Philosophen seien in ihrer Mehrheit stalinistische Betonköpfe gewesen. Es ist wiederum paradox und wurde zuerst von verletzten ostdeutschen Seelen bemerkt, daß die Aburteilungs- und Ausgrenzungsstrategien. die für SED-konformes Verhalten im allgemeinen typisch waren, heute im westlichen Verhalten wieder wirksam werden.

Umgekehrt sind die Verdächtigungen, die von DDR-Philosophen heute gegen die an .,ihre" Universitäten berufenen westdeutsch sozialisierten Nachfolger und (im Ausnahmefall) Kollegen gehegt werden. situationsbedingt bloße Ressentiments, die zum Teil aus dem alten Feindbild-Denken stammen und zum Teil durch neue Erfahrungen motiviert sind (Entlassung. Herabstufung). Die Fähigkeit zur analytischen Kritik war in der DDR kein Bildungsziel; wer sie dennoch erlernte, ist auch gegenwärtig meist frei von Ressentiments.

Es kann heute nicht darum gehen, Gerüchten mehr Gewicht beizumessen, als sie sowieso schon haben. Vielmehr wird von dem wechselseitigem Mißtrauen. für das sich so viele engagieren, gerade überdeckt, wie ähnlich die Verhaltensweisen auf beiden Seiten sind, wie sich die universitätsphilosophischen Kulturen ähneln. Das wissenschaftliche Arbeiten gehorchte vor 199() in beiden Teilen Deutschlands zwar unterschiedlichen Bedingungen. kam jedoch in einer gewissen Akademisierung überein. in einer gewissen Ritualisierung der schriftlichen und mündlichen Vortrags- und Diskussionsformen. So bildete sich auf beiden Seiten eine Unfähigkeit zur Vermittlung von Philosophie außerhalb der Universitäten heraus. Zwar können die vielfältigen ...propagandistischen Aktivitäten“. an denen DDR-Philosophen teilnahmen, als eine Form des Eingehens auf nicht-studentische Zuhörer gelten - als organisierte und Zuhörern wie Dozenten als Pflicht auferlegte Übung war hier allerdings ein genuin philosophisches Interesse an Vermittlung sicher nicht leitend. Heute mag eine solche Unfähigkeit zum Verlassen des akademischen Gehäuses bei einer spezialisierten Wissenschaft unvermeidlich sein, als Charakteristikum einer ganzen Disziplin, die in fast allen ihren Richtungen auf den gesunden Menschenverstand Bezug nimmt. erscheint sie bedenklich. Akademisierung heißt Professionalisierung, und diese hat selbstverständlich die marxistisch-leninistischen Philosophen auf ganz andere Inhalte schwören lassen als die nicht-marxistisch-leninistischen Philosophen. Professionalisierung heißt aber auch Konzentration der individuellen Kommunikationskompetenz auf wenig mehr als das für die Karriere notwendige Maß. und dieser Umstand hat dazu geführt. daß die Universitätsphilosophen aus Ost- und Westdeutschland nach 199() so unglaublich große Schwierigkeiten hatten (und haben), einander zu verstchen. Nicht nur das System der Referenzen (Personen, Schriften. Theorien) war unterschiedlich. auch die Weise des Bezugs (im Ver- hältnis von expliziten und impliziten Bemerkungen. in Zitaten. Fußnoten usw.). So komm es, daß noch heute manche der in der DDR sozialisierten Kollegen keine richtige Anschauung davon haben. wie individuell verschieden sich ihre Westkollegen verstehen (obwohl es philosophische Richtungen und sogar Schulen gibt), während auf der anderen Seite viele BRD-sozialisierte Kollegen kaum einen Begriff davon haben, wie verschiedenen Präferenzen und Interessen sich ihre Ostkollegen zuordnen (obwohl hier alle einer philosophischen Richtung angehörten). Sicher war es nach 199() zuerst ein Unterschied im Wissen. der ein wechselseitiges Verständnis erschwerte: es scheint aber eine beiderseits durchaus ähnliche Unfähigkeit des Umgangs mit ..fremdem“ Wissen zu sein, die dieses Verständnis immer noch verzögert.

Wenn die Philosophen, die an den seit 199() neubesetzten Lehrstühlen in den neuen Bundesländern lehren, sich so wenig für die Philosophie an ihren eigenen Institutionen vor 1990) interessieren, dann liegt das unter anderem daran, daß sie es überhaupt kaum vermögen. sich für die unmittelbare Vergangenheil der Philosophie zu interessieren. Dieses Desinteresse, das alles einschließt, was die soziale und kulturelle Dimension der Philosophie als akademischer Disziplin ausmacht, hat eine Reihe von mehr oder weniger merkbaren Veränderungen begleitet - anders gesagt: Veränderungen sind nicht reflektiert worden. Das trifft auch für die DDR-Philosophen zu, deren Bemühungen um Anpassung verständlicherweise keine Kritik hat laut werden lassen. Was die fachphilosophische Diskussion betrifft, läßt sich an den Beiträgen der ..Deutschen Zeitschrift für Philosophie“ aus den letzten vier Jahren die ..Wende“ in der Interessenlage beobachten: Die Pluralisierung der philosophischen Idiome vollzieht sich ohne Krach und Streit: mit dem Inspizienten hat sich die Souffleuse von der großen Bühne zurückgezogen, und die Akteure reden drauflos, ohne sich sehr darum zu sorgen, ob noch Zuschauer geblieben sind. Wenn hier die Anpassung vollends gelingt, wird auch diese Zeitschrift den Weg zum Fachlich-Sachlichen finden und wie andere Fachorgane vor allem die zu Lesern haben. die in ihr schreiben.

Die Provinzialisierung der deutschen Philosophie in einzelnen ..Szenen“-Hermeneutik, Diskurs- und Kommunikationstheorie, Analytische Philosophie (mit vielen Solisten), ..Französischer Einfluß“, Geschichte der Philosophie (als Genre am verbreitetsten), Phänomenologie, Logik und Argumentationstheorie usw. - greift jetzt auch in den neuen Bundesländern um sich. Wenige Personen und noch weniger Institutionen (vom Kolloquium bis zur thematischen Tagung) überbrücken die sachlich und manchmal auch geographisch auseinanderliegenden Schauplätze des philosophischen Diskurses in Deutschland. Zwar sind die deutschen Philosophen durchaus assoziationsfreudig-die ..Allgemeine Gesellschaft für Philosophie in Deutschlands“ zählt über 700, die ..Gesellschaft für Analytische Philosophie" fast 400 Mitglieder -, sie ringen sich aber nie zu gemeinsamen Aktionen durch.

Das zeigt sich auch bei der wirklich durchgreifenden Veränderung der universitären Definition der Philosophie, wie sie sich in den letzten zehn Jahren zuerst in der BRD, seit 1990) in ganz Deutschland vollzogen hat: der Etablierung der Philosophie als schulisches Unterrichtsfach. Es wird heute eine Entwicklung vollständig rückgängig gemacht, die im 19. Jahrhundert zur Ausgliederung der Philosophie aus den deutschen Schulen geführt hatte (im Gegensatz etwa zu Frankreich. wo Philosophie seit cbenso langer Zeit Schulfach ist). Philosophieprofessoren werden in Deutschland am Ende des 2). Jahrhunderts immer stärker ..Lehrer von Lehrern“. was sie jahrhundertelang hauptsächlich nicht waren. Diese neue Bestimmung ist zugleich höchst variantenreich: Je nach Bundesland sehen die Lehrpläne für 
das Schulfach Philosophie sehr unterschiedlich aus. wie auch die Ausbildungspläne der Lehrer. In einigen Ländern ist Philosophie nur Teil eines Faches ..Ethik". das auch sehr unterschiedlich definiert ist - von einer Moralkunde, die dem im Westen traditionellen Religionsunter richt nahe ist. bis zum interdisziplinär angelegten Bildungsgang.

Es sind zwar überall Kommissionen am Werk gewesen, die auch mit Philosophen besetzt waren, in denen in Zusammenarbeit mit den Ministerien Lehrpläne und Curricula ausgearbeitet und Inhalt wie Form der Schulfächer definiert werden mußten. Eine größere Aufmerksamkeit findet die neue .Funktion” der Universitätsphilosophie aber nicht: Professoren und Assistenten haben. so weit ich sehe, noch kaum als Herausforderung begriffen, daß ..Lehrer"-Studenten neben den ...Magister"-Studenten in den Seminaren sitzen. Die enorme Multiplikationswirkung, der ganz neue und weitgestreute Verbreitungseffekt philosophischen Wissens. das noch viele vor nicht allzu langer Zeit für so schwierig hielten. daß sie es der Wissenschaft vorbehalten wollten - all diese Neuerungen finden so gut wie keinen Eingang in das Selbstverständnis des deutschen Philosophieprofessors, der noch vor kurzem viel Wissenschaftstheorie getrieben hat - als Reflex seiner rein inneruniversitären Rolle. Die in der DDR sozialisierten Philosophen haben hier den gleichen blinden Fleck wie ihre in der BRD sozialisierten Kollegen: Sie scheinen es nicht gelernt zu haben. die konkreten Vermittlungsformen von Philosophie zu reflektieren. Für die Zukunft steht zu befürchten. daß die Philosophen die Definition ihrer gesellschaftlichen Identität bald an jene abgeben, die nach ihnen verlangen: neben den Kultusministerien v. a. Expertenkommissionen. Verlage. Privatpersonen. Hier gibt es bereits ein gewisses Angebot durch akademische Aussteiger, die - ähnlich wie Therapeuten - eine ..Philosophische Praxis" eröffnet haben.

Vielleicht aber zwingt die neue Aufgabe. Lehrer auszubilden, die Philosophieprofessoren dazu, sich in die Köpfe der Schüler zu versetzen und sich über diesen (Um-)Weg der gesellschaftlichen Realität zu nähern. Doch viel Hoffnung darf man wohl nicht haben, daß die radikale Umformung des Faches Philosophie an der Universität auch in neuer Aufmerksamkeit und neuer Verantwortung denen gegenüber resultiert. die dem Anspruch der Wissenschaftlichkeit gemäß bisher nicht berücksichtigt werden mußten. Auch läßt sich derzeit kaum ausmachen. daß die Studenten ihre Interessen gegenüber den institutionalisierten Köpfen vertreten, die so wenig geübt sind. gesellschaftliche Veränderungen wahrzunehmen.

\section{Schlußbemerkung}

Die Situation der Philosophie ist in ganz Deutschland die Situation eines akademischen Fachs an den Universitäten. In den neuen Bundesländern haben die Universitäten zudem - wie die ganze Gesellschaft - ihre politische Struktur vollkommen verändert. Daß diese Veränderung von den Philosophieprofessoren schweigend akzeptiert wurde. erweist sie alle, in Ost und West, als gute Staatsbürger und disziplinierte Wissenschaftler, zu deren Wissenschaft es offenbar nicht mehr gehört, Probleme einer zwar friedlichen, aber radikalen Revolution aller Verhältnisse, gedanklich und im Hinblick auf das eigene Tun zu begleiten. Der nächste Kongreß der ,.Allgemeinen Gesellschaft für Philosophie in Deutschland”. zu dem 1996 in Leipzig ca. 1000) Teilnehmer erwartet werden, bietet im geplanten Programm nirgends ein Forum für Philosophen, die gesellschaftspolitische Lage, geschweige denn ihre eigene Situation zu erörtern. Von der ..Dynamik des Wissens und der Werte“ (so der Titel des Kongresses) nehmen sich die professionellen Denker of fensichtlich selber aus.
Wahrscheinlich rührt die Unfähigkeit zur Selbstreflexion daher, daß die Philosophie in Deutschland als universitäre Institution auf ihr Verhülthis zum Staat verwiesen war und ist, und ihr Verhältnis zur Gesellschaft nur darüber reflektieren kann. Daß aber selbst von dieser Möglichkeit wenig Gebrauch gemacht wird und also über die gesellschaftliche Rolle der Philosophie in Deutschland so unglaublich wenig geschrieben und gestritten wird, hat nicht nur mit der professoralen Abhängigkeit von der Regierung zu tun, sondern wahrscheinlich auch mit der philosophischen Kultur, die eine fast rein akademische, wissenschaftliche Kultur ist. Die deutschen Philosophieprofessoren haben seit 1945 die gesellschaftspolitische Einmischung und Auseinandersetzung so gut wie nie gesucht (Ausnahmen bestätigen auch hier die Regel), und sie sind in dieser Abstinenz vom Aktuellen durch die Ereignisse seit 1990 nicht irritiert worden. Die immerhin gelegentlich geäußerte offene Selbstkritik von DDR-Philosophen bleibt eine einsame Übung, aus der sich für die Zukunft nicht viel lernen läßt. Der Diskurs über „.Nation“ und .,das Nationale“. von wenigen älteren West Professoren zaghaft begonnen. scheint eher Symptom als der Versuch einer Einlassung auf neue Erfahrungsrealitäten. Indem das große Ganze thematisiert wird, kann die eigene Situation ausgeblendet bleiben.

Dr: Ulrich Johannes Schneider, Universität Leipzig, Institut für Philosophie, Augustusplatz 9,04109 Leipzig

\section{Literatur}

Gerd Irrlitz. Ankunft der Utopie, in: Sinn und Form 5. 199(). 93()-955.

Heiner Wilharm. Denken für eine geschlossene Welt. Philosophie in der DDR. Hamburg 199().

Norbert Kapferer. Das Feindbild der marxistisch-leninistischen Philosophie in der DDR 1945-1988. Darmstadt 1990).

Volker Caysa/Petra Caysa/Klaus-Dieter Eichler/Elke Uhl. ..Hoffnung kann entläuscht werden“. Ernst Bloch in Leipzig. Franklurt am Main 1992.

Hans-Peter Krüger. ... Rückblick auf die DDR der 7)er und 8()er Jahre“. in: ders.. Demission der Helden. Berlin 1992. 79-1(1).

Norbert Kapferer (Hg.). Innenansichten ostdeutscher Philosophen. Darmstadt 1994.

Pirmin Stckeler-Weithofer. Philosophic in Leiprig: Neubeginn oder Kontinuität?, in: Hochschule Ost. August 1993. 46-51.

Sonja Brentjes/Peer Pasternak. Berufungsverfahren Ost: Der Spagat zwischen Selbst- und Fremdergänzung. in: Hochschule Ost. Juli/August 1994. 28-43.

Guntolf Herzberg. Erkenntnis und operatives Interesse. Wie die Stasi die akademische Philosophic einspannte und aushorchte (Vortrag auf dem Deutschen Historikertag in Leipzig). in: Frankfurter Allgemeine Zeitung vom 30. 9.1 994. 42.

\section{Anmerkung der Redaktion}

Wir stellen den Beitrag von Ulrich Johannes Schneider hiermit zur Diskussion. Unsere LeserInnen möchten wir ausdrücklich ermuntern, sich in Leserbriefen mit den aufgeworfenen Problemen auseinanderzusetzen. In Heft 2/1996 werden wir erste kritische Stellungnahmen zum Text publizieren. 